\title{
The Effect of Continuous Suspension Constraint on the Free Vibration and Buckling of a Beam
}

\author{
Róbert K. Németh ${ }^{1 *}$, Bilal M. A. Alzubaidi ${ }^{1}$ \\ ${ }^{1}$ Department of Structural Mechanics, Faculty of Civil Engineering, Budapest University of Technology and Economics, H-1521 \\ Budapest, P.O.B. 91, Hungary \\ * Corresponding author, e-mail: nemeth.robert@emk.bme.hu
}

Received: 29 January 2021, Accepted: 10 May 2021, Published online: 14 May 2021

\begin{abstract}
In this paper, the free vibration and the buckling of a continuously suspended simply-supported beam are analyzed. A semi-analytical approach is used to calculate the natural circular frequencies and the critical forces of the beam. The length of the suspension is used as a parameter, and the natural circular frequencies and the critical forces are presented in a frequency map or a buckling map. The maps are analyzed in view of the trivial solutions, and the frequency map is compared to the map of discrete cable-stayed beams. Finally, for the validation of the results a numerical, finite element analysis is performed.
\end{abstract}

Keywords

block-and-tackle suspension, frequency map, buckling map

\section{Introduction}

Block-and-tackle suspension of cable-stayed beams [1], and other structural elements [2] offer a new way for supporting structural elements in multiple points with only a slight increase in the static indeterminacy of the structure. In these constraints, a cable is driven through a series of pulleys, constraining the total displacements of the supported points. Fig. 1(a) and (b) show the possible topology of the suspension cable with two and four suspension points, respectively. The red arrows represent the oneparameter dependent resultants of the cable force acting on the structure, while the sum of the supported points' displacements must be zero if the elongation of the cable is neglected. The effect of this support on the structural response is analyzed in [3], and the dynamical analysis was performed in $[1,4]$ with emphasis on the possible slacking of the cable and the resulting nonlinear vibration. For a beam, it is easy to construct a double suspension system on both sides of the beam, which then eliminates the chaotic motion of the beam as one of the cables would always be in tension while the other would be slacked.

In [5], families of beams were analyzed, where the number and the position of the suspension points were varied, and the linear natural circular frequencies were calculated with the assumption of the cables to be in tension. The resulting frequency map is a diagram of the frequency
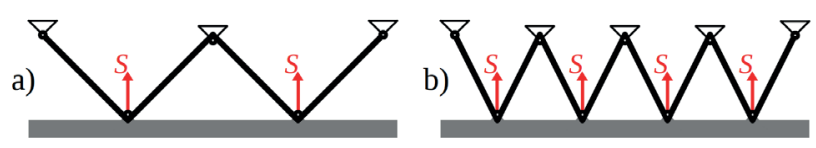

c)

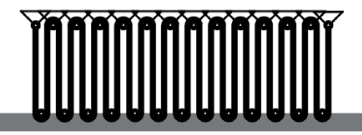

Fig. 1 Block-and-tackle suspension. Suspension cable and cable reactions a) with two suspension points; b) with four suspension points; c) with infinite suspension points

parameters as a function of a continuous topological parameter (the position of the suspension points). It offers a good overview of the effect of the position of the suspension point on the vibration of the structure. The increase of the number of the discrete suspension points leads as a limit case to a continuous suspension, where the total displacement of the suspended segment (the integral of the displacement function) will be zero. At the same time, the "cable" exerts a universal distributed load on the beam (see Fig. 1(c)). The intensity of the distributed load is proportional to the amplitude of the integral of the displacements.

The universal distributed reactions are not common in the mechanical modelling, but it is not impossible. Considering the elastic supported beams and their Pasternakmodel [6] with a rigid shear layer, the soil-reaction has 
a constant value, when a given segment of the soil is in compression, so, the reaction does not depend on the magnitude of the points' own displacements. However, that model differs from the model we introduce in this paper: in the continuous suspension cable model the reactions act on the uplifting points as well, and the intensity is proportional to the displacements' amplitude. Beyond the theoretical derivation of the above constraint, it can be originated from another model too. The continuously suspended segment could be connected to a fluid vessel, which contains in-compressible fluid, resulting in a zero total displacement and a constant pressure on the segment.

In this paper, we aim to analyze the effect of continuous support on the buckling and the free vibration properties of a beam. As the suspension cable is attached to the simply supported beam; it affects the natural circular frequencies, the buckling forces, the vibration modes, and the buckling modes. This effect will be analyzed as a function of the length of the suspended segment. The natural circular frequencies or the critical forces of the beam are calculated as a function of the length of the continuous support and presented in a frequency map or a buckling map, respectively. The structure of the paper is the following: in Section 2, we will present the mechanical model and the parametric formulation of the buckling and the frequency matrix. In Section 3, the applied numerical method for calculating the buckling and frequency map is presented. In Section 4, we present the calculated maps and analyze them regarding their limits, trivial modes. In Section 5, we validate our calculation against an FE-analysis performed in a commercial FE-software. Finally, in Section 6, we summarize the results and draw our conclusions.

\section{Mechanical model}

\subsection{Model parameters and governing equations}

The investigated beam's mechanical model is shown in Fig. 2. The beam of length $L$ is an Euler-Bernoulli-beam. We assume small displacements; the principal displacement is the $w(x)$ translation of the beam axis. The material's Young's modulus is $E$ and the cross-sectional moment

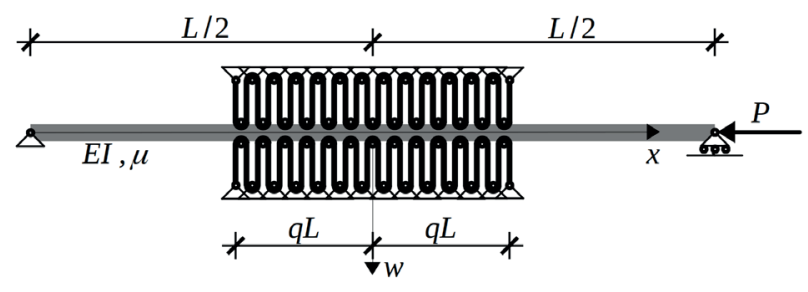

Fig. 2 The analyzed beam with a continuous cable support of inertia is $I$. The mass density is denoted by $\rho$, the specific mass of the beam is denoted by $\mu$. In the dynamic analysis we neglect the rotary inertia of the cross-section. The beam is supported by a pinned joint on its left end and by a roller on its right end. The beam is divided into three segments, and their displacements are denoted by $w_{1}(x)$, $w_{2}(x)$, and $w_{3}(x)$, respectively. The $1^{\text {st }}$ and $3^{\text {rd }}$ segments refer to the free parts of the beam while the $2^{\text {nd }}$ segment is suspended by infinitely dense continuous, unstretchable cables, one on the bottom side and one on the top side. The continuous suspension extends symmetrically at a distance $q L$ from the midpoint of the beam in both directions. Thus, $2 q$ can be referred to as the supported ratio of the length of the beam. The supporting effect of the cable is such that the total displacement of the second segment of the beam must be zero: slacking of the cable on any side would involve the elongation of the cable on the other side. At the same time, the cables exert a universal distributed load of intensity $S_{0}$. We refer to this resultant intensity as cable force, but we note here, that $S_{0}$ could be negative as well, the sign of $S_{0}$ represents the pulled side of the beam. Thus, the two-sided cable support eliminates the nonlinearity of the structure, which would be caused by the slacking of a one-sided suspension. The mass of the cable and any friction are neglected. For the stability analysis the axial force $P$ is acting on the right end of the beam, as presented in Fig. 2.

The origin of the reference system is set to the midpoint of the beam. This positioning is required to postpone numerical problems during the calculation of the determinant of the frequency matrix. The $q$ ratio runs between $1 / 1000$ and $1 / 2$. For the numerical calculations, we used the $L=1$ value, but we generalized the results everywhere.

For the dynamic study, the beam in Fig. 2 is assumed to obey the Euler-Bernoulli dynamic beam equation [7]. For the free segments, the equations are:

$$
\begin{aligned}
& E I w_{1}^{\prime \prime \prime \prime}+\mu \ddot{w}_{1}=0, \\
& E I w_{3}^{\prime \prime \prime \prime}+\mu \ddot{w}_{3}=0 .
\end{aligned}
$$

Here, and from now on, prime (') denotes the partial derivation with respect to the $x$ position, while the dot (') denotes the partial derivation with respect to the time $t$. For the $2^{\text {nd }}$ segment, the dynamic beam equation is:

$$
E I w_{2}{ }^{\prime \prime \prime \prime}+\mu \ddot{w}_{2}=S(t) .
$$

For the buckling analysis, the $4^{\text {th }}$ order static differential equation of the Euler-Bernoulli beam applies with the 
application of the compression force [8]. The equations for the unsupported beam segments are:

$$
\begin{aligned}
& E I w_{1}^{\prime \prime \prime \prime}+P w_{1}^{\prime \prime}=0, \\
& E I w_{3}^{\prime \prime \prime \prime}+P w_{3}^{\prime \prime}=0 .
\end{aligned}
$$

For the $2^{\text {nd }}$ segment, the differential equation contains the cable force as well:

$$
E I w_{2}^{\prime \prime \prime \prime}+P w_{2}^{\prime \prime}=S_{0} \text {. }
$$

In both the dynamic and the buckling problem, the nontrivial solution consists of $w_{1}(x), w_{2}(x)$, and $w_{3}(x)$ functions, which fulfill the respective differential equations, namely Eqs. (1)-(3) for the dynamic problem and Eqs. (4)-(6) for the buckling problem. The general solutions of the homogeneous differential equations contain 4-4 parameters while the solutions of the non-homogeneous differential equations have 4-4 parameters from the general solution of the complementary homogeneous equation and one parameter from the particular solution.

In each case, there are a total of 13 parameters which must be related to each other such that they fulfill the following 13 constraints. There are 2-2 boundary conditions at the ends of the beam (the translation and the bending moment, thus the curvature of the beam must be zero there):

$w_{1}(-L / 2)=w_{3}(L / 2)=0$

$w_{1}^{\prime \prime}(-L / 2)=w_{3}^{\prime \prime}(L / 2)=0$.

There are four-four connection conditions at the boundaries of the segments (the translation, rotation, bending moment, and shear force must be the same from both segments):

$$
\begin{aligned}
& w_{1}(-q L)=w_{2}(-q L), w_{2}(q L)=w_{3}(q L), \\
& w_{1}^{\prime}(-q L)=w_{2}^{\prime}(-q L), w_{2}^{\prime}(q L)=w_{3}^{\prime}(q L), \\
& w_{1}^{\prime \prime}(-q L)=w_{2}^{\prime \prime}(-q L), w_{2}^{\prime \prime}(q L)=w_{3}^{\prime \prime}(q L), \\
& w_{1}^{\prime \prime \prime}(-q L)=w_{2}^{\prime \prime \prime}(-q L), w_{2}^{\prime \prime \prime}(q L)=w_{3}^{\prime \prime \prime}(q L),
\end{aligned}
$$

Finally, the scaling parameter of the particular solution connects the cable force intensity and the amplitude of the displacement function through the continuous constraint:

$$
\int_{-q L}^{q L} w_{2} d x=0
$$

We present the above equations on the specific problems separately in the following subsections.

\subsection{Derivation of the frequency matrix}

For the dynamic analysis we assume harmonic vibration of the beam with a natural circular frequency of $\omega_{0}$. Thus, we can separate the variables in the solution of the differential equations Eqs. (1)-(3) and we assume the solution in the form:

$$
\begin{aligned}
& w_{1}(x, t)=w_{1}(x) \cos \left(\omega_{0} t\right), \\
& w_{2}(x, t)=w_{2}(x) \cos \left(\omega_{0} t\right), \\
& w_{3}(x, t)=w_{3}(x) \cos \left(\omega_{0} t\right) .
\end{aligned}
$$

At the same time, the cable force intensity changes in time with the same harmonic function:

$$
S(t)=S_{0} \cos \left(\omega_{0} t\right) \text {. }
$$

We note here, that the $w_{1}(x), w_{2}(x), w_{3}(x)$ functions and $S_{0}$ together specify the assumed vibration mode. There is no unique nontrivial solution for them: if multiply the amplitude of the vibration by an $\alpha$ scalar parameter, then $w_{1}(x), w_{2}(x), w_{3}(x)$ functions and $S_{0}$ must be multiplied by the same $\alpha$. We also remind the reader, that small displacements and the two-sided suspension allows $S_{0}$ to take up negative values too.

After the substitution of the assumed solutions into the differential equations, simplification by $\cos \left(\omega_{0} t\right)$ yields the ordinary differential equations for the three segments:

$$
\begin{aligned}
& E I w_{1}^{\prime \prime \prime \prime}-\mu \omega_{0}^{2} w_{1}=0, \\
& E I w_{2}^{\prime \prime \prime \prime}-\mu \omega_{0}^{2} w_{2}=S_{0}, \\
& E I w_{3}^{\prime \prime \prime \prime}-\mu \omega_{0}^{2} w_{3}=0 .
\end{aligned}
$$

For the homogeneous equations, we have the following general solutions:

$$
\begin{aligned}
w_{1}(x)= & A_{1} \sin (\lambda x)+A_{2} \cos (\lambda x)+A_{3} \sinh (\lambda x)+ \\
& A_{4} \cosh (\lambda x), \\
w_{3}(x)= & A_{9} \sin (\lambda x)+A_{10} \cos (\lambda x)+A_{11} \sinh (\lambda x)+ \\
& A_{12} \cosh (\lambda x) .
\end{aligned}
$$

In the above equations, $\lambda$ is the frequency parameter. Using the general solutions in the differential equations show that it is related to the natural circular frequency as: $\omega_{0}=\lambda^{2} \sqrt{E I / \mu}$.

For the $2^{\text {nd }}$ segment, a particular solution is 
$w_{2}^{p}(x)=\frac{S_{0}}{\mu \omega_{0}^{2}}=\frac{S_{0}}{E I \lambda^{4}}$,

while the general solution with the solution of the complementary equation is

$$
\begin{aligned}
w_{2}(x)= & A_{5} \sin (\lambda x)+A_{6} \cos (\lambda x)+A_{7} \sinh (\lambda x)+ \\
& A_{8} \cosh (\lambda x)+\frac{S_{0}}{E I \lambda^{4}} .
\end{aligned}
$$

In the above three general solutions, there are altogether 13 parameters, $A_{1}$ through $A_{12}$ in addition to $S_{0}$, which are determined from the boundary and continuity conditions listed in Eqs. (7)-(13). The integration in the last condition yields:

$$
A_{6} \sin (\lambda q L)+A_{8} \sinh (\lambda q L)+S_{0} \frac{q L}{E I \lambda^{3}}=0 .
$$

Substituting the general solutions and their respective derivatives into the constraints yields a homogeneous system of linear equations in the form:

$$
\boldsymbol{F} \boldsymbol{a}=0,
$$

where the vector $\boldsymbol{a}$ contains the unknown parameters:

$$
\boldsymbol{a}=\left[\begin{array}{llllll}
A_{1} & A_{2} & \ldots & A_{11} & A_{12} & \frac{S_{0}}{E I}
\end{array}\right]^{T},
$$

while the $\boldsymbol{F}$ is the frequency matrix built from the coefficients of the parameters. After a simplification of the rows of the matrix by the lambda multipliers, where applicable, the matrix can be written in the following block structure:

$$
\boldsymbol{F}=\left[\begin{array}{cccc}
\boldsymbol{B}_{n} & \mathbf{0}_{4} & \mathbf{0}_{4} & 0 \\
\boldsymbol{C}_{n} & -\boldsymbol{C}_{n} & \mathbf{0}_{4} & -\boldsymbol{s} \\
\mathbf{0}_{4} & \boldsymbol{C}_{p} & -\boldsymbol{C}_{p} & \boldsymbol{s} \\
\mathbf{0}_{2} & \mathbf{0}_{2} & \boldsymbol{B}_{p} & 0 \\
\mathbf{0}_{1} & \boldsymbol{D} & \mathbf{0}_{1} & q L / \lambda^{3}
\end{array}\right],
$$

where the $\boldsymbol{B}$ and $\boldsymbol{C}$ matrices belong to the boundary and continuity conditions, respectively, the $n$ and $p$ indices denote the evaluation at a negative or positive $x$-value, the vector $\boldsymbol{s}$ is the first unit vector divided by $\lambda^{4}$, the $\mathbf{0}_{j}$ matrices are $j \times 4$ zero-matrices. Using the odd and even properties of the functions, the entries of the non-zero matrices are:

$$
\begin{aligned}
& \boldsymbol{B}_{n}=\left[\begin{array}{cccc}
\sin \left(\frac{\lambda L}{2}\right) & \cos \left(\frac{\lambda L}{2}\right) & -\sinh \left(\frac{\lambda L}{2}\right) & \cosh \left(\frac{\lambda L}{2}\right) \\
\sin \left(\frac{\lambda L}{2}\right) & -\cos \left(\frac{\lambda L}{2}\right) & -\sinh \left(\frac{\lambda L}{2}\right) & \cosh \left(\frac{\lambda L}{2}\right)
\end{array}\right], \\
& \boldsymbol{B}_{p}=\left[\begin{array}{cccc}
\sin \left(\frac{\lambda L}{2}\right) & \cos \left(\frac{\lambda L}{2}\right) & \sinh \left(\frac{\lambda L}{2}\right) & \cosh \left(\frac{\lambda L}{2}\right) \\
-\sin \left(\frac{\lambda L}{2}\right) & -\cos \left(\frac{\lambda L}{2}\right) & \sinh \left(\frac{\lambda L}{2}\right) & \cosh \left(\frac{\lambda L}{2}\right)
\end{array}\right] . \\
& \boldsymbol{C}_{n}=\left[\begin{array}{cccc}
-\sin (\lambda q L) & \cos (\lambda q L) & -\sinh (\lambda q L) & \cosh (\lambda q L) \\
\cos (\lambda q L) & \sin (\lambda q L) & \cosh (\lambda q L) & -\sinh (\lambda q L) \\
\sin (\lambda q L) & -\cos (\lambda q L) & -\sinh (\lambda q L) & \cosh (\lambda q L) \\
-\cos (\lambda q L) & -\sin (\lambda q L) & \cosh (\lambda q L) & -\sinh (\lambda q L)
\end{array}\right], \\
& \boldsymbol{C}_{p}=\left[\begin{array}{cccc}
\sin (\lambda q L) & \cos (\lambda q L) & \sinh (\lambda q L) & \cosh (\lambda q L) \\
\cos (\lambda q L) & -\sin (\lambda q L) & \cosh (\lambda q L) & \sinh (\lambda q L) \\
-\sin (\lambda q L) & -\cos (\lambda q L) & \sinh (\lambda q L) & \cosh (\lambda q L) \\
-\cos (\lambda q L) & \sin (\lambda q L) & \cosh (\lambda q L) & \sinh (\lambda q L)
\end{array}\right], \\
& \boldsymbol{D}=\left[\begin{array}{llll}
0 & \sin (\lambda q L) & 0 & \sinh (\lambda q L)] .
\end{array}\right.
\end{aligned}
$$

The nontrivial solution requires a singular $\boldsymbol{F}$ matrix, where its determinant is zero. The solutions of this nonlinear equation yield the frequency parameters, then the ratios of the 13 parameters can be obtained from Eq. (27).

\subsection{Derivation of the stability matrix}

For the stability analysis, the general solutions of the homogeneous equations Eqs. (4)-(5) are:

$$
\begin{aligned}
& w_{1}(x)=B_{1} \sin (k x)+B_{2} \cos (k x)+B_{3} x+B_{4}, \\
& w_{3}(x)=B_{9} \sin (k x)+B_{10} \cos (k x)+B_{11} x+B_{12} .
\end{aligned}
$$

In the above equations, $k$ is the load parameter. Using the general solutions in the differential equations show that it is related to the compression force as:

$k^{2}=\frac{P}{E I}$.

The particular solution of the non-homogeneous equation Eq. (6) is

$$
w_{2}^{p}(x)=\frac{S_{0} x^{2}}{2 P}=\frac{S_{0} x^{2}}{2 E I k^{2}},
$$


and so the general solution is:

$$
w_{2}(x)=B_{5} \sin (k x)+B_{6} \cos (k x)+B_{7} x+B_{8}+\frac{S_{o} x^{2}}{2 E I k^{2}} \text {. }
$$

Similarly to the dynamic analysis, there are altogether 13 parameters in the above three general solutions, $B_{1}$ through $B_{12}$ in addition to $S_{0}$, which are determined from the boundary and continuity conditions listed in Eqs. (7)-(13). The integration in the last condition yields:

$B_{6} \frac{\sin (k q L)}{k}+B_{8} q L+\frac{S_{0}(q L)^{3}}{6 E I k^{2}}=0$.

Substituting the general solutions and their respective derivatives into the constraints yields a homogeneous system of linear equations in the form:

$\boldsymbol{S b}=0$.

where the vector $\boldsymbol{b}$ contains the unknown parameters:

$\boldsymbol{b}=\left[\begin{array}{llllll}B_{1} & B_{2} & \ldots & B_{11} & B_{12} & \frac{S_{0}}{E I}\end{array}\right]^{T}$,

while the $\boldsymbol{S}$ is the frequency matrix built from the coefficients of the parameters. After a simplification of the rows of the matrix by the lambda multipliers, where applicable, the matrix can be written in the following block structure:

$\boldsymbol{S}=\left[\begin{array}{cccc}\boldsymbol{B}_{n} & \mathbf{0}_{4} & \mathbf{0}_{4} & 0 \\ \boldsymbol{C}_{n} & -\boldsymbol{C}_{n} & \mathbf{0}_{4} & \boldsymbol{s}_{n} \\ \mathbf{0}_{4} & \boldsymbol{C}_{p} & -\boldsymbol{C}_{p} & \boldsymbol{s}_{p} \\ \mathbf{0}_{2} & \mathbf{0}_{2} & \boldsymbol{B}_{p} & 0 \\ \mathbf{0}_{1} & \boldsymbol{D} & \mathbf{0}_{1} & (q L)^{3} /\left(6 k^{2}\right)\end{array}\right]$,

where the $\boldsymbol{B}$ and $\boldsymbol{C}$ matrices belong to the boundary and continuity conditions, respectively, the $n$ and $p$ indices denote the evaluation at a negative or positive $x$-value, the $\mathbf{0}_{j}$ matrices are $j \times 4$ zero-matrices. Using the odd and even properties of the functions, the entries of the non-zero matrices are:
$\boldsymbol{B}_{n}=\left[\begin{array}{cccc}-\sin \left(\frac{k L}{2}\right) & \cos \left(\frac{k L}{2}\right) & \frac{-L}{2} & 1 \\ \sin \left(\frac{k L}{2}\right) & -\cos \left(\frac{k L}{2}\right) & 0 & 0\end{array}\right]$,
$\boldsymbol{B}_{p}=\left[\begin{array}{llll}\sin \left(\frac{k L}{2}\right) & \cos \left(\frac{k L}{2}\right) & \frac{L}{2} & 1 \\ -\sin \left(\frac{k L}{2}\right) & -\cos \left(\frac{k L}{2}\right) & 0 & 0\end{array}\right]$,

$\boldsymbol{C}_{n}=\left[\begin{array}{cccc}-\sin (k q L) & \cos (k q L) & -q L & 1 \\ \cos (k q L) & \sin (k q L) & 1 / k & 0 \\ \sin (k q L) & -\cos (k q L) & 0 & 0 \\ -\cos (k q L) & -\sin (k q L) & 0 & 0\end{array}\right]$,

$\boldsymbol{C}_{p}=\left[\begin{array}{cccc}\sin (k q L) & \cos (k q L) & q L & 1 \\ \cos (k q L) & -\sin (k q L) & 1 / k & 0 \\ -\sin (k q L) & -\cos (k q L) & 0 & 0 \\ -\cos (k q L) & \sin (k q L) & 0 & 0\end{array}\right]$,

$\boldsymbol{D}=\left[\begin{array}{llll}0 & \frac{\sin (\lambda q L)}{k} & 0 & q L\end{array}\right]$,

$\boldsymbol{s}_{n}=\left[\begin{array}{c}-(q L)^{2} /\left(2 k^{2}\right) \\ q L / k^{3} \\ -1 / k^{4} \\ 0\end{array}\right], \quad \boldsymbol{s}_{p}=\left[\begin{array}{c}(q L)^{2} /\left(2 k^{2}\right) \\ q L / k^{3} \\ 1 / k^{4} \\ 0\end{array}\right]$.

The nontrivial solution requires a singular $\boldsymbol{S}$ matrix, where its determinant is zero. The solutions of this nonlinear equation yield the load parameters, then the ratios of the 13 parameters can be obtained from Eq. (37).

\section{Numerical method used for the calculation}

Both the free vibration and the buckling problem requires the formulation of the determinant of a $13 \times 13$-matrix and finding those parameter values, where it will be equal to zero. For the calculation we fixed the $q$ parameter in 500 points between $1 / 1000$ and $1 / 2$. For each different case we increased the $\lambda$ or the $k$ parameter from zero upward to a maximum of 60 in 10000 steps and calculated the determinant numerically from the analytical form in Eq. (29) or Eq. (39). When the sign of the determinant changed between two parameter values, then the position of the zero value was interpolated between the two values of opposite sign, and a solution for the frequency parameter or the load parameter was found, and we stored the first 18 of them for each $q$ value. 
As results will show, both the vibration modes and the buckled modes are well separated: the curves of the subsequent $\lambda$ or $k$ parameters remain in a clearly visible distance from each other at any $q L$ suspension length, i.e., the neighboring curves do not cross each other and do not get close to a crossing. As it was shown in [5], with discrete number of suspension points there were such crossings, which also yielded the disturbance of the order of the symmetric and skew-symmetric modes. The lack of crossings is why such a simple scanning is sufficient for this problem instead of the more robust methods [9].

\section{Numerical results}

\subsection{Results of the dynamic analysis}

First, we present the frequency map of the beam in Fig. 3. One can notice that starting from the bottom until the $11^{\text {th }}$ line, the odd modes have constant lines, representing constant frequency values of the odd modes while the line of frequencies of even modes are curved. Furthermore, there are strange zigzagged thin lines appearing for higher modes starting from the higher $q$ values. This type of solutions is known from the literature of numerical scanning of parameter spaces [10-11]: the reason is that the function value, which is interpolated to find its zero, cannot be calculated accurately at the current parameter level. Because of that, the numerical results of the mathematical problem are not the real solution of the physical problem. Hence, these lines are also referred to as parasitic solutions. While calculating the frequency map of Fig. 3., higher $\lambda q$ products as arguments of the sinh and cosh functions caused exponential increase of several terms in the determinant of the $\boldsymbol{F}$ matrix. Due to the accumulation of truncation errors in that order of magnitude, even the sign of the determinant became inaccurate. Thus, the chaotic backand-forth change of the sign of the determinant caused the vast number of the parasitic solutions in the top right corner of the diagram. In our further analysis we will not consider those solutions.

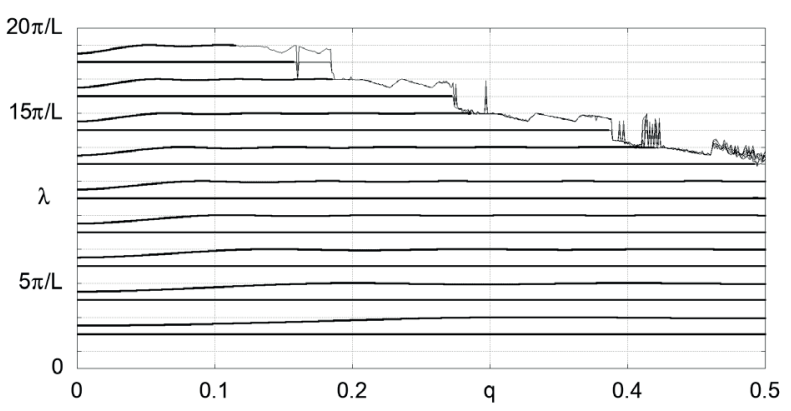

Fig. 3 Frequency map of the analyzed beam
The constant value of the lines of the odd modes is easy to explain. These modes are the skew-symmetric sinusoidal vibration modes with $A_{1}=A_{5}=A_{9}$, while every other parameter $\left(A_{\mathrm{m}}\right.$-s and $\left.S_{0}\right)$ are zero, and the frequency parameter is

$\lambda_{2 j-1}=2 j \pi / L$.

Thus, for the $(2 j-1)^{\text {th }}$ mode $w_{1}(x)=w_{2}(x)=w_{3}(x)=A_{1}$ $\sin (\lambda x)$, and as such, it naturally fulfills the conditions in Eqs. (9)-(13). The boundary conditions of Eqs. (7)-(8) are fulfilled because of the specific value of the frequency parameter according to Eq. (41).

The even modes are the symmetric vibration modes, where $A_{1}=-A_{9}, A_{2}=A_{10}, A_{3}=-A_{11}, A_{4}=A_{12}, A_{5}=0$, and $A_{7}=0$. For better visibility of the even modes, we zoomed into the frequency map and presented these modes separately in Fig. 4 (a)-(d). It can be seen that the curved line of the $j^{\text {th }}$ even mode never exceeds the

$\lambda_{2 j}^{\max }=(2 j+1) \pi / L$

value and it reaches the $\lambda_{2 j}^{\max }$ value at exactly $j$ values of $q$, namely at the

$q_{j}^{l}=l /(2 j+1), l=1, \ldots, j$

points. These modes are special too, with $A_{2}=A_{6}=A_{10}$, and every other parameter is zero, resulting in a sinusoidal mode of $2 j+1$ half-waves. So, with $w_{1}(x)=w_{2}(x)=w_{3}(x)=A_{2}$ $\cos (\lambda x)$, it naturally fulfills the conditions in Eqs. (9)-(13). The boundary conditions of Eqs. (7)-(8) are fulfilled because of the specific value of the frequency parameter according to Eq. (42).

To highlight the similarity between the curved lines of the even modes in Fig. 4, we made the following transformation on them: we subtracted from the $\lambda_{2 j}$ values $(2 j+1)$ $\pi / \mathrm{L}$, while along the $q$ axis each curve was stretched by $2 j+1$. The resulting diagram is shown in Fig. 5 . The domain of definition is different for the curves: the first one ends at 1.5 , the second one at 2.5 , etc., but we can observe a
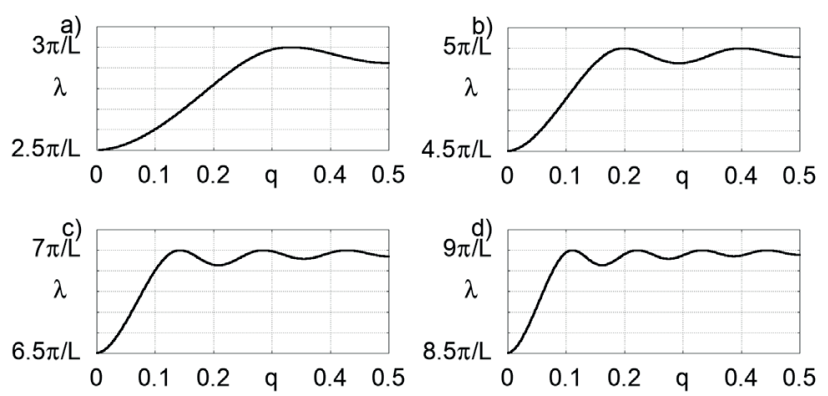

Fig. 4 Details of the frequency map of the analyzed beam. a) $2^{\text {nd }}$ mode, b) $4^{\text {th }}$ mode, c) $6^{\text {th }}$ mode, d) $8^{\text {th }}$ mode 


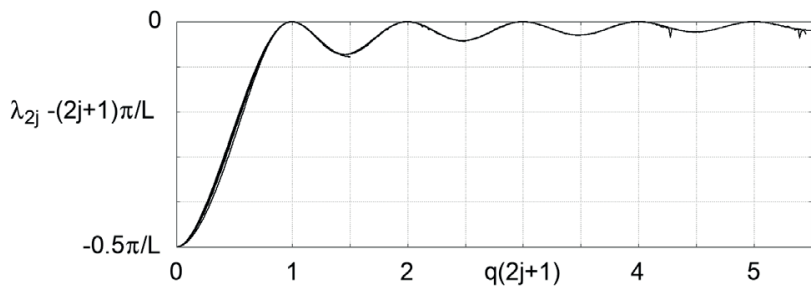

Fig. 5 Curves of even modes of the frequency map transformed into overlapping curves

skeleton of the curves with maximums at integer values and minimums near the half points between the integer ones. The skeleton is followed by each curve until their last segment of the length 0.5 . In these last segments the curves are slightly below the skeleton.

There is a limit case where $q$ tends toward zero. This value mechanically corresponds to a girder supported by three simple support. The frequency parameters of this structure can be obtained from the literature [7] and they show a good agreement.

We compared the calculated frequency map with those of beams suspended in discrete points. Maps of the discrete structures were taken from [5] and an equivalent $q$ parameter was calculated for the discrete structures such that it equals the total horizontal projections of the sloped cables. In Fig. 6, we present the results for the even modes (odd modes are skew-symmetric in each case and result in the constant diagram as Eq (41) predicts). Red, green, blue, cyan, magenta, and brown lines are the maps corresponding to 2-4-6-8-10-12 suspension points, respectively, while black lines are regular (non-parasitic) solution points of the continuous suspension. Colored lines below the black ones represent that the given number of suspension points is insufficient to model the continuous suspension. In those cases, the frequency parameter is smaller, and some of them even go below the $\lambda_{2 j-1}$ value of the "previous" odd mode resulting in crossings, which is not the case in the continuous suspension. Dashed thin hyperbolas are drawn to specify the sinusoidal solutions according to Eq. (43). It can be seen that the discrete suspension regularly skips some of those sinusoidal mode's frequency parameters.

The application of the results is quite straightforward. Based on the $q$ parameter and the required number of modes we are interested in, either Eq. (41) can be used for the odd modes, or we can read out an initial value for the frequency parameter from Fig. 5. Then, the frequency parameter must be substituted into Eq. (27) and solve for the $\boldsymbol{a}$ vector, while the natural circular frequency can be calculated from Eq. (23).

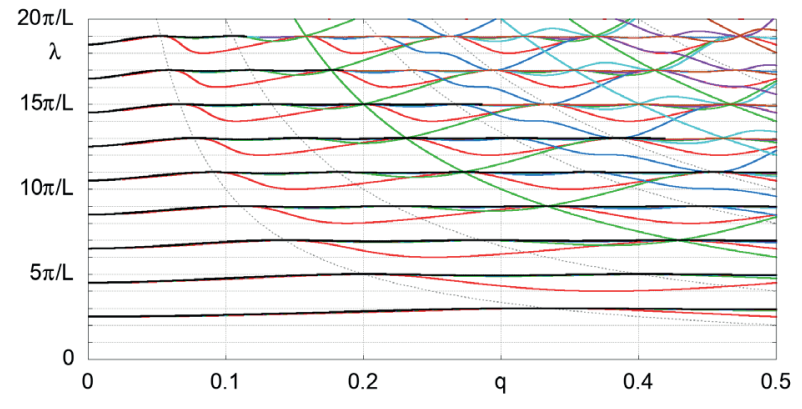

Fig. 6 Convergence of the frequency map of the family of beams suspended in discrete points to that of the continuous suspended beam

\subsection{Results of the buckling analysis}

Next, we present the buckling map of the beam in Fig. 7. Due to the structure of the differential Eqs. (4)-(6), there are no hyperbolic terms in the general solutions, so no parasitic solutions appear on the map. Similarly to the frequency map, starting from the bottom, the odd modes have a constant value while the load parameter lines of even modes are slightly curved. The curves of the even modes vary in a much narrower domain, than in Fig. 3., but Fig. 8 reveals that the curves of the even modes are not straight lines.

The constant value of the lines of the odd modes is explained the same way as earlier. These modes are the skew-symmetric sinusoidal buckling modes with $B_{1}=B_{5}=B_{9}$, while every other parameter $\left(B_{\mathrm{m}}-\mathrm{s}\right.$ and $\left.S_{0}\right)$ are zero, and the load parameter is

$k_{2 j-1}=2 j \pi / L$.

Thus, for the $(2 j-1)^{\text {th }}$ mode $w_{1}(x)=w_{2}(x)=w_{3}(x)=B_{1}$ $\sin (k x)$, and as such, it naturally fulfills the conditions in Eqs. (9)-(13). The boundary conditions of Eqs. (7)-(8) are fulfilled because of the specific value of the load parameter according to Eq. (44).

The even modes are the symmetric buckling modes, where $B_{1}=-B_{9}, B_{2}=B_{10}, B_{3}=-B_{11}, B_{4}=B_{12}, B_{5}=0$, and $B_{7}=0$. For better visibility of the even modes, we zoomed

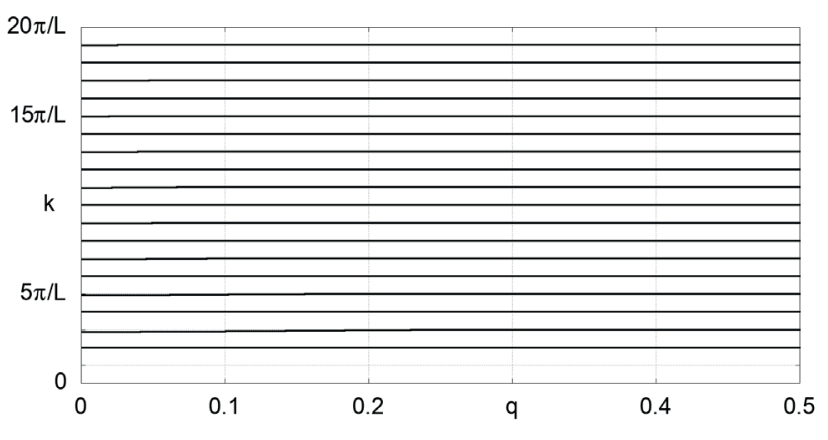

Fig. 7 Buckling map of the analyzed beam 

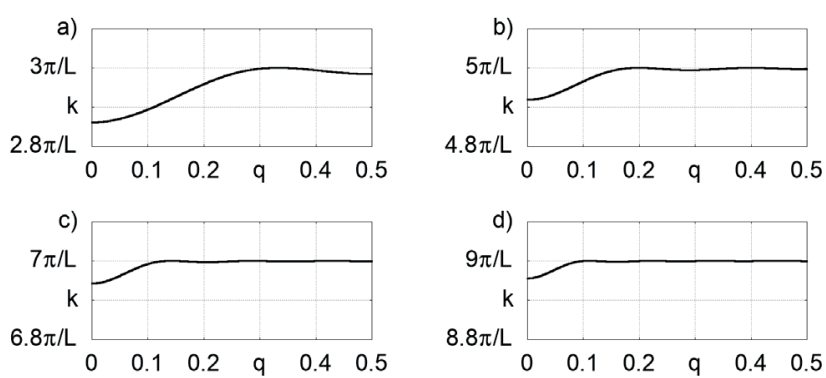

Fig. 8 Details of the buckling map of the analyzed beam. a) $2^{\text {nd }}$ mode, b) $4^{\text {th }}$ mode, c) $6^{\text {th }}$ mode, d) $8^{\text {th }}$ mode

into the buckling map and presented them separately. In Fig. 8. (a)-(d). It can be seen that the curved line of the $j^{\text {th }}$ even mode never exceeds the

$k_{2 j}^{\max }=(2 j+1) \pi / L$

value and it reaches the $k_{2 j}^{\max }$ value at exactly $j$ values of $q$, namely at the

$q_{j}^{l}=l /(2 j+1), l=1, \ldots, j$

points. (Note: these are the same structural parameters that appear in Eq. (43).) These modes are special too, with $B_{2}=B_{6}=B_{10}$, and every other parameter is zero, resulting in a sinusoidal shape of $2 j+1$ half-waves. So, with $w_{1}(x)=w_{2}(x)=w_{3}(x)=B_{2} \cos (k x)$, it naturally fulfills the conditions in Eqs. (9)-(13). The boundary conditions of Eqs. (7)-(8) are fulfilled because of the specific value of the load parameter according to Eq. (45).

To highlight the similarity between the curved lines of the even modes in Fig. 8, we made the following transformation on them: we subtracted from the $k_{2 j}$ values $(2 j+1) \pi / L$ and then multiplied by $2 j+1$, while along the $q$ axis each curve was stretched by $2 j+1$. The resulting diagram is shown in Fig. 9. The domain of definition is different for the curves: the first one ends at 1.5, the second one at 2.5, etc., but we can observe a skeleton of the curves with maximums at integer values and minimums near the half points between the integer ones. The skeleton is followed by each curve until their last segment of the length 0.5 . In these last segments the curves are slightly below the skeleton.

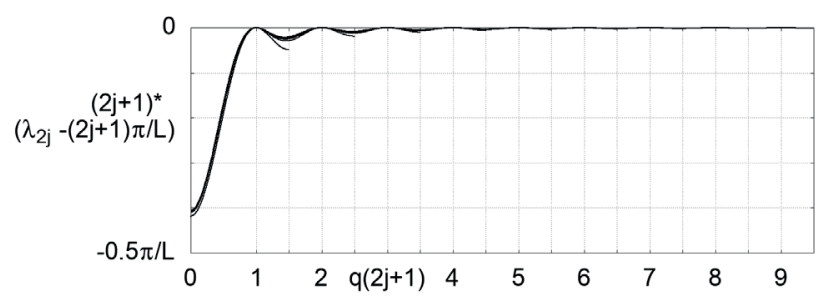

Fig. 9 Curves of even modes of the buckling map transformed into overlapping curves
The limit case, where $q$ tends toward zero, can be checked here as well. This value mechanically corresponds to a girder supported by three simple supports. The load parameters of this structure can be obtained from the literature [8], and they show a good agreement.

The application of the results is quite straightforward. Based on the $q$ parameter and the required number of shapes we are interested in, either Eq. (44) can be used for the odd modes, or we can read out an initial value for the load parameter from Fig. 9. Then, the load parameter must be substituted into Eq. (37) and solve for the $\boldsymbol{b}$ vector, while the critical load can be calculated from Eq. (33).

\section{Validation of the results}

\subsection{Development of the FE-model}

To validate our semi-analytical results, we compared the calculated natural circular frequencies and critical loads to those results calculated in the commercial finite element software ANSYS for a member of the analyzed family with $q=0.25$. As the continuous constraint is not presented in the software, we substituted it with a swing system, which allows the constraint of the sum of the displacements of multiple points, while the transmitted internal reaction forces are the same. The latter is the reason why swing systems are used in experiments to simulate distributed loads. The basis of the swing system is a beam with two bars at its end and one in its middle in the opposite lateral direction, as shown in Fig. 10(a). The displacement of the mid-point is the average of the displacements of the ends of the rigid beam, while the equilibrium of the rigid beam yields the same force in the end-bars. The elementary segments are connected consecutively to the analyzed beam and to each other, and the last bar is supported by a simple support, as an example shows in Fig. 10(b). In the resulting beam, the sum of the displacements of the supported points remains zero. Unlike the cables, the bars are capable of resisting compression forces as well, so there is no need to apply a swing system on both sides of the beam, because already a one-sided suspension yields a linear structure. Our numerical analysis showed that simple bar elements could be used to model the beams of the swing, a)

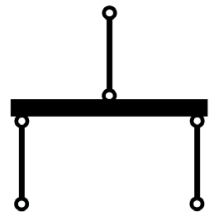

b)

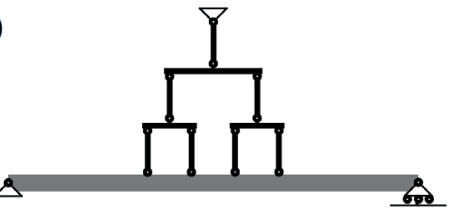

Fig. 10 Swing system for the discrete modeling of the supporting effect of the cable: a) a single swing-element, b) a compiled swing system 
so we were using that simpler model. We also note here that to avoid rigid body like motion; the beams must be supported in the horizontal direction, but for easier understanding, we did not draw those supports.

In the ANSYS model, we used BEAM188 elements for the modeling of the beam itself. The swing system consisted of massless LINK180 elements with the same cross-section but higher Young's modulus. In the numerical model, the rigidity is often approximated with a numerically sufficiently large stiffness, which yields similar results to the actual structure. So, before further computations, we performed convergence analysis of the following two components of the numerical model.

- the number of elements of the analyzed beam,

- the normal stiffness of the bars in the swing system.

Fig. 11 shows the convergence of the second natural frequency and the second buckling force of the beam as one of the above two parameters was varied (the other was held at the finally accepted value). As a conclusion, we will use 1024 elements for the discretization of the beam, and Young's modulus of the link elements will be 1280 times the Young's modulus of the beam.
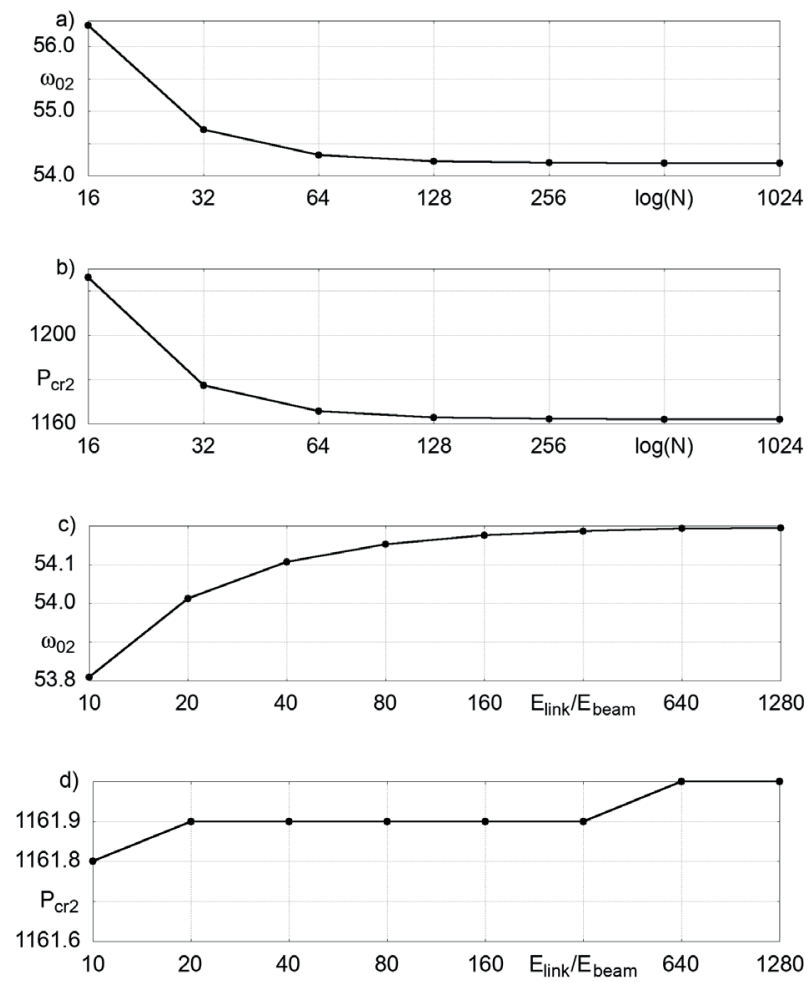

Fig. 11 Results of the convergence analysis of the model. Horizontal axis (logarithmic scale): number of beam elements $(a, b)$ ratio of stiffnesses (c, d). Vertical axis: second natural frequency (a, c) second critical load (b, d)

\subsection{Numerical results from the FE-model}

In the ANSYS model, we used the following material properties and geometry. The cross-section was chosen to be a square, with a side length of $2 \mathrm{~cm}$. Thus, the area was $4 \mathrm{~cm}^{2}$, and the inertia $1.333 * 10^{-8} \mathrm{~m}^{4}$. The Young's modulus was chosen to $109 \mathrm{~N} / \mathrm{m}^{2}$ yielding a bending stiffness of $13.33 \mathrm{Nm}^{2}$. The mass density was $2000 \mathrm{~kg} / \mathrm{m}^{3}$, yielding a specific mass of $0.8 \mathrm{~kg} / \mathrm{m}$. The length of the beam is $L=1 \mathrm{~m}$.

We computed the natural circular frequencies and the critical forces with a series of swing systems, where the number of bars connected to the beam was $n=4,8,16$, $\ldots, 256$. The bars were arranged symmetrically, and the distance between two neighboring bars at the beam was $2 q L / n$.

Fig. 12(a) shows the ratio of the first 10 computed natural circular frequencies to the semi-analytical results obtained from Fig. 3 as a function of the number of discrete suspension points. Red, green, blue, and magenta lines correspond to the $2^{\text {nd }}, 4^{\text {th }}, 7^{\text {th }}$, and $9^{\text {th }}$ natural frequencies, respectively, which are converging to the continuous case a bit slower, but even the worst case has less than $7 \%$ error. Other modes align with the 1.00 line.

Fig. 12 b) shows the ratio of the first 10 computed critical forces to the semi-analytical results from Fig. 7 as a function of the number of discrete suspension points. Here, the deviation from 1.00 is even smaller; red, green, blue, cyan, and magenta lines correspond to the $2^{\text {nd }}, 4^{\text {th }}$, $6^{\text {th }}, 8^{\text {th }}$, and $10^{\text {th }}$ critical forces.

Finally, we compare two vibration modes, namely the second mode computed with 256 suspension points. Fig. 13(a) shows the semi-analytically calculated mode

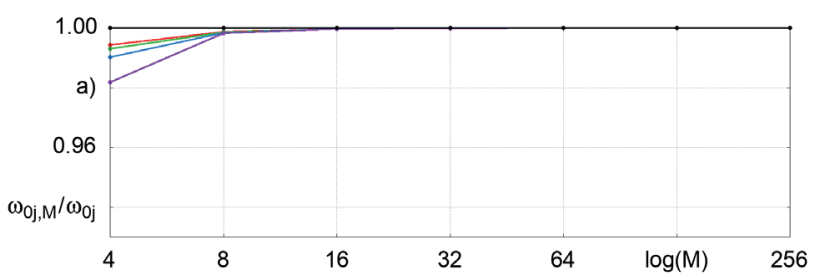

(a)

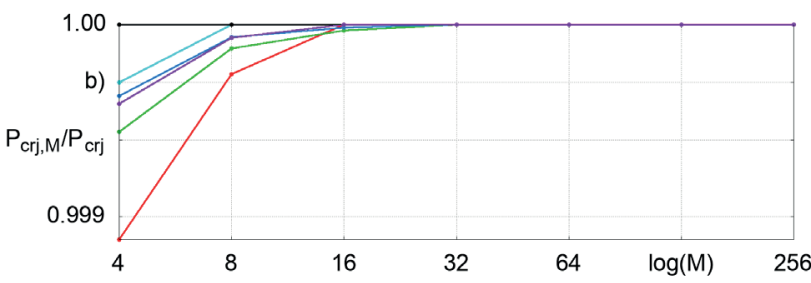

(b)

Fig. 12 Convergence with the number of discrete suspension points of a) the ratios of the natural circular frequencies, and b) the ratios of the critical forces 


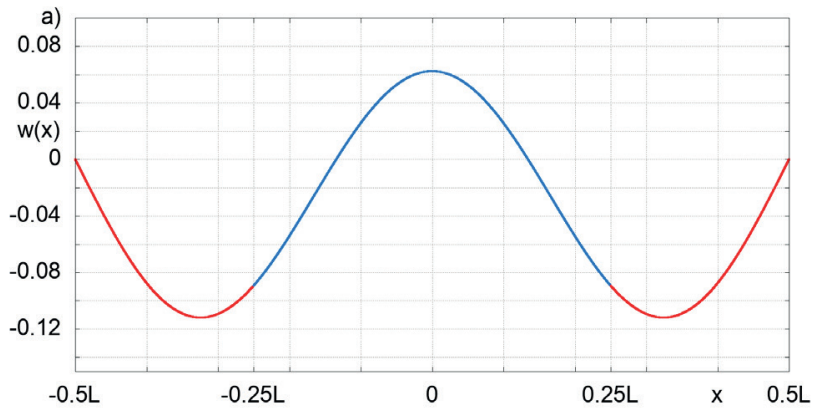

(a)

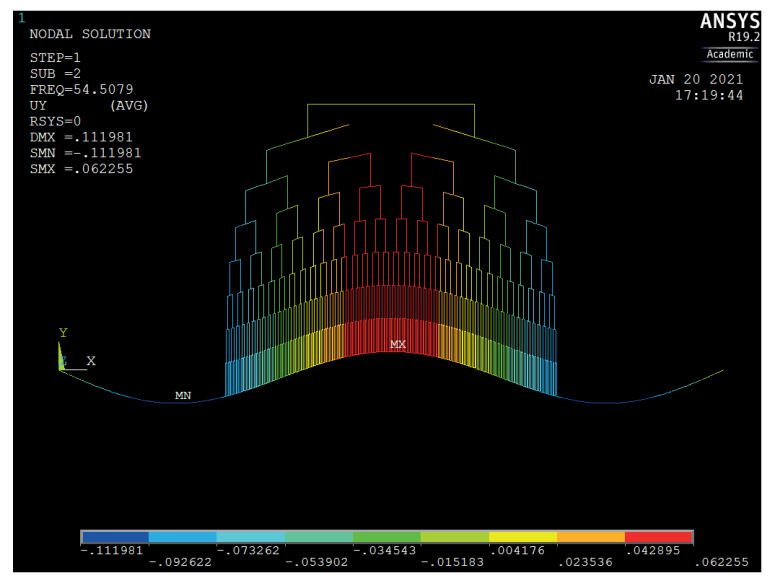

(b)

Fig. 13 The second vibration mode of the beam with $q=0.25$ : a) alculated with the semi-analytical method (blue curve is the suspended segment, red curve is the non-suspended segment), b) computed with ANSYS (colors represent the displacements)

with the three functions in Eqs. (21)-(22) and Eq. (25) (the suspended segment is highlighted with a different color), while Fig. 13(b) shows the vibration mode calculated with ANSYS. The modes are scaled to the same minimum of -0.112 downward and have the same maximum value of 0.062 upward and show a good agreement. Furthermore, the $S_{0}$ distributed cable force from Eq. (27) will be $3291 \mathrm{~N} / \mathrm{m}$ in the semi-analytical model while the reaction of the last bar is $1642 \mathrm{~N}$ from ANSYS, which must be

\section{References}

[1] Kocsis, A., Németh, R. K., Turmunkh, B. "Dynamic analysis of a beam on block-and-tackle suspension system: A continuum approach", Engineering Structures, 101, pp. 412-426, 2015. https://doi.org/10.1016/j.engstruct.2015.07.022

[2] Hincz, K. "Arch-supported tensile structures with very long clear spans", Journal of the International Association for Shell and Spatial Structures, 48(154), pp. 89-98, 2007.

[3] Hincz, K. "Nonlinear Analysis of Cable Net Structures Suspended from Arches with Block and Tackle Suspension System, Taking into Account the Friction of the Pulleys", International Journal of Space Structures, 24(3), pp. 143-152, 2009.

https://doi.org/10.1260/026635109789867643 distributed over the $2 q L$ length, which yields a distributed cable force of $3284 \mathrm{~N} / \mathrm{m}$. The two intensities have less than a $2.2 \%$ difference, which is an acceptable difference.

\section{Conclusions}

In this paper, we presented the free vibration and the buckling analysis of a simply supported beam suspended by a continuous constraint, which can be considered as a limit case of the block-and-tackle suspension system with a discrete number of suspension points. From the analytical derivation of the frequency matrix and the stability matrix, the frequency map and the buckling map were numerically calculated.

We analyzed the frequency map and the buckling map and presented a simple method for the approximate calculation of the frequency parameters and the critical load parameters, which are the basis of the natural circular frequencies and the critical forces, respectively. We gave a formula for those parameters of the length of the suspension, where a specific vibration mode becomes a sinusoidal shape.

We compared the frequency map to the convergence of the discrete suspended beams and validated our results against results of an FE-model calculated with the commercial software ANSYS.

The frequency map and the buckling map offer a quick method for the approximate calculation of natural circular frequencies and critical forces while their numerical calculation did not involve the solution of a large generalized eigenvalue problem as it is usual when an FE-model is used for the computation.

\section{Acknowledgment}

This work was supported by the Hungarian National Research, Development and Innovation Office (NKFIH): Grant Nr. 119440, Grant Nr. 128584; TKP2020 NC, Grant No. BME-NCS.

[4] Németh, R. K., Geleji, B. B. "Nonlinear Normal Modes of a Piecewise Linear Continuous Structure with a Regular State", Periodica Polytechnica Civil Engineering, 62(4), pp. 1039-1051, 2018.

https://doi.org/10.3311/PPci.12512

[5] Németh, R. K., Geleji, B. "Függesztett gerendacsalád szabadrezgése II.: Frekvenciatérkép számítása és vizsgálata, (Free vibration of a family of suspended beams II. Calculation and analysis of the frequency map)", Építés - Építészettudomány, 46(3-4), pp. 351-370, 2018. (in Hungarian) https://doi.org/10.1556/096.2017.006 
[6] Kerr, A. D. "On the formal development of elastic foundation models", Ingenieur-Archiv, 54, pp. 455-464, 1984. https://doi.org/10.1007/BF00537376

[7] Weaver, W., Timoshenko, S. P., Young, D. H. "Vibration Problems in Engineering", John Wiley \& Sons, New York, NY, USA, 1990.

[8] Timoshenko, S. P., Gere, J. M. "Theory of Elastic Stability", Dover Publications, Mineola, NY, USA, 2009.

[9] Domokos, G., Szeberényi, I. "A hybrid parallel approach to nonlinear boundary value problems", Computer Assisted Mechanics and Engineering Sciences, 11, pp. 15-34, 2004.
[10] Németh, R. K. "Parallel scanning of implicit surfaces with the simplex algorithm", Periodica Polytechnica Civil Engineering, 58(1), pp. 23-31, 2014.

https://doi.org/10.3311/PPci.7032

[11] Gáspár, Zs., Domokos, G., Szeberényi, I. "A parallel algorithm for the global computation of elastic bar structures", Computer Assisted Mechanics and Engineering Sciences, 4, pp. 55-68, 1997. 